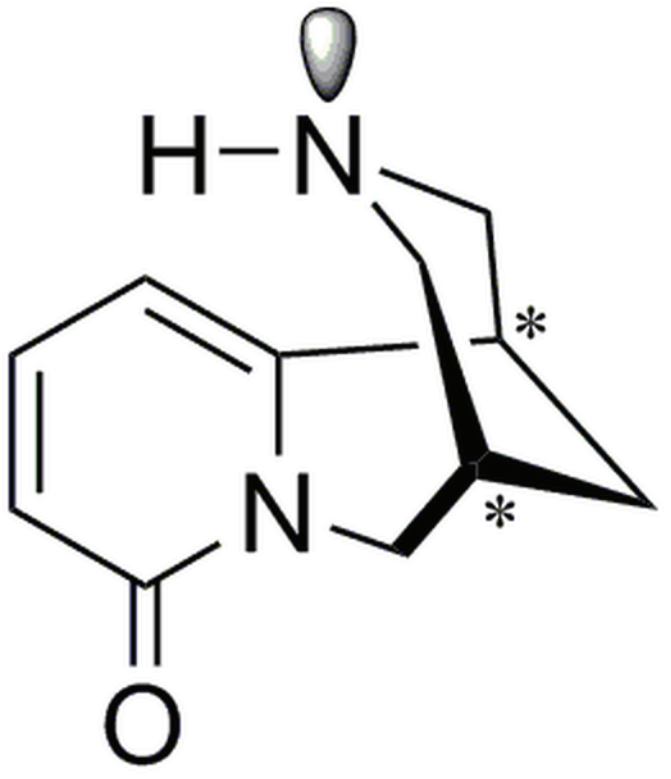

Figure 1. Scheme of (-)Cytisine

Keywords: cytisine, salts, electron density, topological analysis

\section{MS28-P8 The influence of data collection quality on the multipolar parameters of sulfur and chlorine atoms in \\ 5-chloro-2-mercaptobenzimidazole \\ (CMBZT) \\ Anita M. Owczarzak ${ }^{1}$, Maciej Kubicki}

\section{Adam Mickiewicz University}

email: aniow@ amu.edu.pl

During our studies of charge density distribution in thioamide compounds, we very often were faced with the evidence of electron density depletion around the nuclei of sulfur atoms, which can be observed as a certain ring in the deformation charge density maps (fig 1.). As such a "ring effect" is observed regularly in charge density studies not only for sulfur but also for other heavier atoms (chlorine, bromine ect.) we decided to look more closely at this subject.

The high resolution data has been collected using Xcalibur diffractometer with Eos detector at $100 \mathrm{~K}$ for two 5-chloro-2-mercaptobenzimidazole (CMBZT) crystals of deliberately different quality,. The structures have been refined using Hansen-Coppens multipolar model [1] implemented in MoPro software [2], using (almost) exactly the same procedure for both cases. In a communication we will focus on a comparison of the multipole parameters and topological analyses of CMBZT compound. In addition, the influence of cutting off the data on the results of refinement has been also studied.

[1]Hansen, Coppens, P.Acta Crystallographica Section A 34, 909-921 (1978).

[2]Jelsch,Guillot, Lagoutte, Lecomte, J. Appl. Cryst. $38,38-54$ 


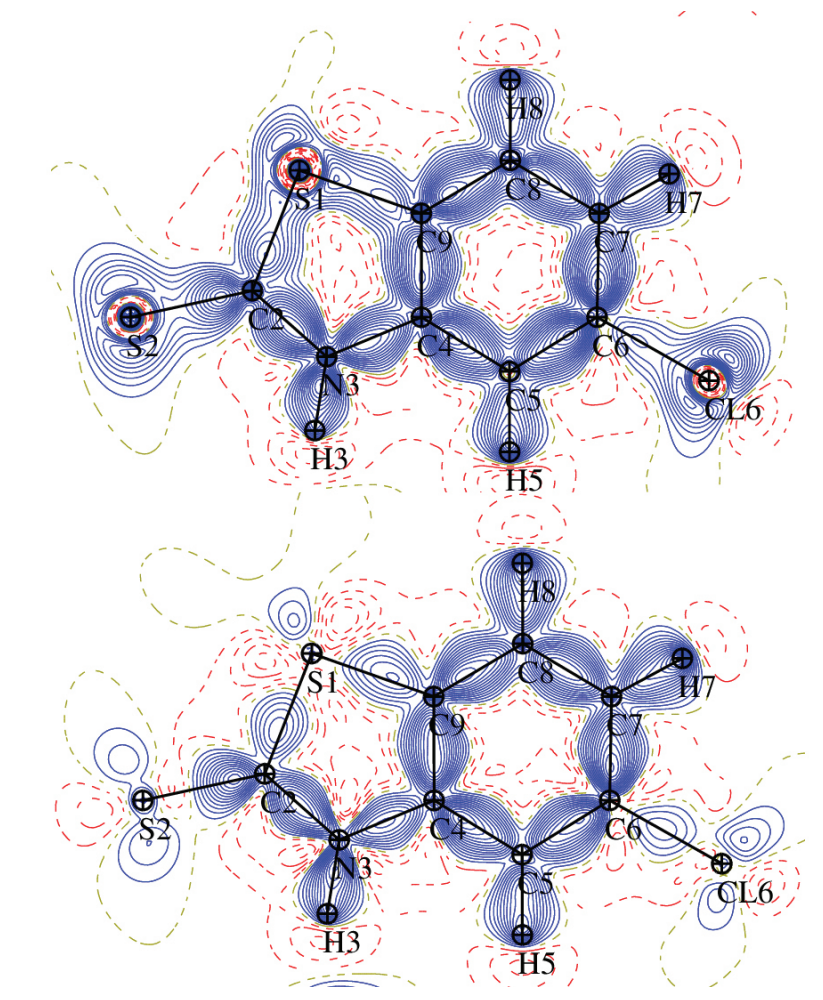

Figure 1. The static deformation density map of CMBZT, obtained from different data.

Keywords: charge density, thioamide, sulfur

\section{MS28-Pg Charge density studies on polymorphic co-crystals}

Lucy K. Mapp ${ }^{1}$, Mateusz B. Pitak ${ }^{1}$, Simon J. Coles ${ }^{1}$, Srinivasulu Aitipamula $^{2}$

1. University of Southampton, University Road, Southampton, SO17 1BJ, UK

2. Institute of Chemical and Engineering Sciences, A*STAR (Agency for Science, Technology and Research), 1 Pesek Road, Jurong Island, 627833, Singapore

email: 1.mapp@soton.ac.uk

Polymorphism is a widely-studied phenomena, and of huge importance especially in pharmaceutical industries, as well as a factor to consider in crystal engineering and solid-state studies. That a compound can exhibit different crystalline structures, often with differing physical properties, is remarkable, as well as the fact that the origin of such behaviour is often unknown. Multi-component systems, too, are widely studied and such systems can also exhibit this behaviour. We present the results of comparative studies of two polymorphic forms of the analgesic drug ethenzamide 1:1 co-crystallised with saccharin. The aim of this work is to further understanding of the differences between the structures and their related properties by investigating the electronic distributions, and the properties thereof, in the solid state.

Two polymorphs of the 1:1 co-crystal of ethenzamide (2-ethoxybenzamide, EA) and saccharin (SAC) have previously been reported [1]. Form I crystallises in the triclinic P-1 space group, whilst form II is monoclinic, $\mathrm{P} 21 / \mathrm{n}$. Both polymorphs display the primary carboxy-amide-imide heterosynthon interaction between molecules. However, it is at the second level of hydrogen bonding, extending the hydrogen bonding network through the structure, where polymorphic variation can be seen. Here extended 1D linear tapes are present in form I, generated via $\mathrm{N}-\mathrm{H} \bullet \bullet \mathrm{O}$ hydrogen bonding interactions, whilst form II exhibits stacked tetrameric motifs which utilise both $\mathrm{N}-\mathrm{H} \bullet \bullet \mathrm{O}$ hydrogen bonds and $\mathrm{C}-\mathrm{H} \bullet \bullet \mathrm{O}$ interactions. Thus, the polymorphs can be classified as synthon polymorphs at the second level of hydrogen bonding.

To investigate these structures further, high-resolution charge density distribution analysis has been carried out to enable differences in electronic structures to be determined and ascertain how these differences relate to the polymorphic behaviour. The Hansen-Coppens formalism [2] and Bader's AIM theory [3] provide a quantitative analysis and determination of both intra- and intermolecular interactions, in terms of their nature and role in these systems.

[1] S. Aitipamula, P. S. Chow, R. B. H. Tan, CrystEngComm., 2009,11, 889-895

[2] N. Hansen, P. Coppens, Acta Cryst. 1978, A34, 909-921

[3] R. F. W. Bader, Atoms in Molecules: A Quantum Theory, Clarendon: Oxford, UK, 1990 